\title{
Statistical Shape Modelling of the Levator Ani with Thickness Variation
}

\author{
Su-Lin Lee ${ }^{1,2}$, Paramate Horkaew ${ }^{1}$, Ara Darzi $^{2}$, and Guang-Zhong Yang ${ }^{1,2}$ \\ ${ }^{1}$ Royal Society/Wolfson Foundation Medical Image Computing Laboratory \\ ${ }^{2}$ Department of Surgical Oncology and Technology \\ Imperial College London, London, UK \\ \{su-lin.lee, paramate.horkaew, a.darzi, g.z.yang\}@imperial.ac.uk
}

\begin{abstract}
The levator ani is vulnerable to injury during childbirth and effective surgical intervention requires full knowledge of the morphology and mechanical properties of the muscle structure. The shape of the levator ani and regional thickening during different levels of physiological loading can provide an indication of pelvic floor dysfunction. This paper describes a coupled approach for shape and thickness statistical modelling based on harmonic embedding for volumetric analysis of the levator ani. With harmonic embedding, the dynamic information extracted by the statistical modelling captures shape and thickness variation of the levator ani across different subjects and during varying levels of stress. With this study, we demonstrate that the derived model is compact and physiologically meaningful, demonstrating the practical value of the technique.
\end{abstract}

\section{Introduction}

The pelvic floor is a complex structure composed of a diaphragm of striated muscle covered by fascia. The levator ani is the part of the pelvic floor for visceral support and for drawing the anal canal upwards and forwards. It is highly susceptible to injuries during natural childbirth, which can lead to problems with urination and defecation [1]. Defective pelvic organ support is one of the most common problems in women. Among them, over $20 \%$ require a second operation. The management of pelvic floor disorders in women is traditionally divided between gynaecologists, urologists and colorectal surgeons. The modern surgical approach to the problem is to perform all necessary repairs during one single operation to avoid the usual territorial subdivision and patient re-operation. This requires an accurate preoperative identification of all prolapsed organs and their mechanical properties.

Magnetic Resonance Imaging (MRI) in both closed and open access scanners has been used to assess any injuries of the levator. Open access (interventional) scanners are becoming popular as the subject sits in an anatomically natural position, where the pelvic floor muscles can optimally exert its full function [2]. Dynamic information can also be acquired by using fast acquisition protocols with which each image can be acquired in less than 2 seconds. Full 3D acquisition of the entire levator ani volume during straining has so far proven to be difficult due to the prolonged imaging time required to recover the fine structural details of the levators. Diagnosis has usually 
been made based on distances to specific landmarks in a single image slice - typically mid-sagittal or mid-coronal [3].

Hoyte et al [4] used 3D reconstructions of the levator ani to observe the differences between symptomatic and asymptomatic subjects. Similar work in the reconstruction of the entire levator ani has been accomplished in other research institutions [5] to compare the levators of symptomatic and asymptomatic subjects. The scans have been limited to levators at rest due to the time constraint for which a subject can maintain a certain dynamic position. Previous work at this institution has investigated the shape variation of the levator ani by using statistical shape modelling of the surface of the levators with harmonic embedding [6]. The training set consisted of levators at rest, maximal contraction and maximal squeeze down with 3D MR data sets acquired using a fast spin echo gradient recalled echo protocol. As the surface of the levator is topologically equivalent to a compact 2D manifold with boundary, the model was constructed based on original work by Horkaew and Yang [7].

Existing research has shown that the strain distribution of the levator ani during different levels of physiological loading plays an important part in assessing its mechanical function. To this end, Hoyte et al have recently extended their work to study the thickness of the levator ani [8]. They have shown that colour thickness mapping of the levator ani can be used for the swift evaluation of a large number of levators. In their study, levators of symptomatic and asymptomatic subjects at rest were manually segmented and then divided into four quadrants. It was found that the right anterior quadrant was thicker in asymptomatic subjects although the significance of this has not yet been determined. This is an indirect way of assessing the strain distribution during stress, and thickness mapping may be performed in a number of ways. The direction along which the thickness is determined may be the direction to a corresponding control point on the opposite surface, the direction to the closest point on the opposite surface or the surface or vertex normal. For more detailed analysis, it is possible to adopt previous work in shape and volume modelling in medical image computing. For example, medial representations [9] can be used to model the thickness of the levator ani. This consists of a set of medial atoms that contain two vectors of equal length, each originating from the atom. A mesh of medial atoms could be used to represent the thickness of a sheet muscle such as the levator ani.

The purpose of this paper is to extend our previous work on optimal statistical shape modelling for volumetric analysis of the levator ani. With harmonic embedding, it provides an effective framework for combining shape with tensor distribution for optimal statistical modelling. We demonstrate that for sheet muscles such as the levator ani, the method can capture meaningful dynamic shape and thickening variations.

\section{Method}

\subsection{Data Acquisition}

For assessing shape and volumetric variations across subjects and during different levels of physiological loading, two training data sets were acquired for this study. The first was built from transverse images acquired from a 1.5T Siemens Sonata closed access scanner. A fast turbo-spin echo non-zone selective protocol was used to 
acquire $T_{2}$ weighted images $(T R=1500 \mathrm{~ms}, T E=130 \mathrm{~ms}$, slice thickness $=3 \mathrm{~mm})$ of the pelvic floor at rest, with the subjects in a supine position. Acquisition time was approximately 5 minutes with 32 slices obtained from each subject with image size $256 \times 168$ pixels and spatial resolution of $1 \times 1 \mathrm{~mm}$. Ten subjects were scanned with this protocol. The second set was built from coronal slices from a $0.5 \mathrm{~T}$ GE iMR open access scanner. A fast turbo spin echo protocol was used to acquire $T_{2}$ weighted images $(\mathrm{TR}=4900 \mathrm{~ms}, \mathrm{TE}=102 \mathrm{~ms}$, slice thickness $=5 \mathrm{~mm})$ of the pelvic floor with the subjects in both sitting and squatting positions. A total of 15 slices were acquired for each subject and the acquisition time was approximately 8 minutes. Whilst a subject is in the squatting position, the shape of the levator ani changed due to the weight bearing down on the levators, and four subjects were scanned with this protocol. All subjects scanned were nulliparous females.

\subsection{Harmonic Shape Embedding}

Similar to our previous study, the levators were manually segmented from the MR images. Each volume was automatically separated into the top and bottom surfaces [10] by determining the points of highest curvature. Each surface was then triangulated, scaled and aligned by using the Iterative Closest Points algorithm [11]. Shape embedding was performed by using the technique previously developed [7], where triangulated and aligned surfaces are harmonically mapped to a unit quadrilateral based domain. A B-spline surface patch was constructed from each mesh by reparameterising the harmonic embedding over uniform knots. The surfaces are reparameterised over the unit base domain with the parameterization defined by a piecewise bilinear map.

\subsection{Incorporating Thickness Variation}

After harmonic shape embedding, the associated muscle thickness $d(p)$ was measured at each control point $p$ by using its minimum distance to the corresponding surface [12]. By using the bottom surface of the levator as the base domain, the surface tangents to the control points on the top surface give a local linear approximation of the top surface. Therefore, the shortest distance between $p$ and the plane $T p$ gives a local thickness measure. That is,

$$
d(p)=\min \operatorname{dist}(p, T p)
$$

To achieve a combined statistical shape and volume modelling, the dimensionality of the original problem of shape modelling needs to be extended to include $d$ described above. For this study, the Minimum Description Length (MDL) criterion was used to determine the optimal control points that can yield the most compact description of the shape and thickness variations. In this case, the corresponding objective function can be written as: 


$$
F=\sum_{m}\left(-\sum_{S} \ln P\left(\left\{\begin{array}{l}
x \\
y \\
z \\
d
\end{array}\right\}_{S}^{m} \mid \sigma_{m}\right)+L\left(\sigma_{m}\right)\right)
$$

where $\left\{\{x, y, z, d\}^{T}\right\}_{s}^{m}$ is the projection of the $s^{\text {th }}$ shape and thickness into the $m^{\text {th }}$ axis of the model whose variance is $\sigma_{m}^{2}$. We assume in this study that the distributions of the coordinates and $d$ are of Gaussian and the model parameters are uniformly distributed. In this case, the log likelihood and length of the model parameters are:

$$
\begin{aligned}
& \ln P\left(x_{s}^{m} \mid \sigma_{m}\right)=\ln \Delta+\frac{1}{2} \ln \left(2 \pi \sigma_{m}^{2}\right)-\frac{1}{2}\left(\left\{\begin{array}{l}
x \\
y \\
z \\
d
\end{array}\right\}_{s}^{m} / \sigma_{m}\right)^{2} \\
& L\left(\sigma_{m}\right)=1+\ln \left(\frac{\sigma_{\text {max }}-\sigma_{\min }}{\delta}\right)+|\ln \delta|
\end{aligned}
$$

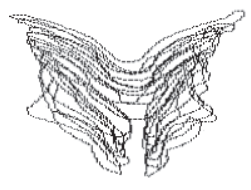

(a)

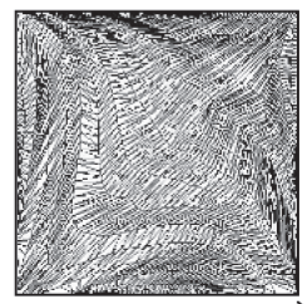

(b)

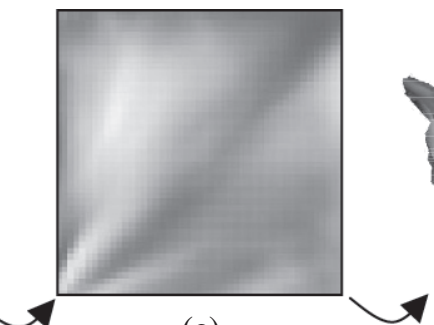

(c)

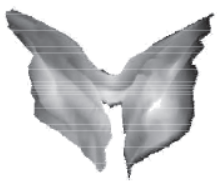

(d)

Fig. 1. (a) A segmented levator ani volume whose bottom surface has been made into a (b) harmonic map and shown here (c) with corresponding thickness map and (d) the thickness map displayed on the bottom surface of the levator ani.

The optimisation was performed by using Polak-Ribiere's conjugate gradient optimisation algorithm [13]. At each iteration, the coordinates of each control point were moved in the harmonic embedded space and the associated thickness was recalculated in the original re-projected 3D shape space by following the local thickness measure described above. Figure 1 schematically summarises the overall computational steps involved in the proposed algorithm, showing the harmonic embedded space used for determining the optimal control points for capturing both shape and thickness variations, and the final modelling results before and after remapping the thickness distribution to the $3 \mathrm{D}$ model of the levator ani. 


\section{Results}

Figure 2 illustrates two example cross sections of the pelvic floor acquired by using the closed and open access scanners respectively, where the structure associated with the levator ani is indicated by the arrows. They generally provide enough resolution for the manual segmentation of the levators. The corresponding training sets used in the study are shown in (c) and (d) where local muscle thickness is mapped by using a colour scale ranging from cool to warm colours ${ }^{1}$. Warmer shades indicate a thicker part of the levator and the maximum scale used is $10 \mathrm{~mm}$.

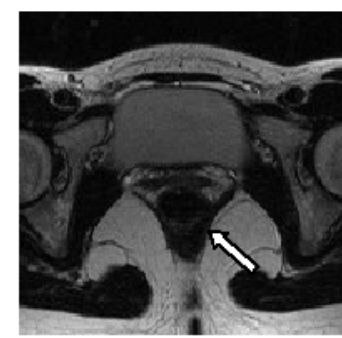

(a)

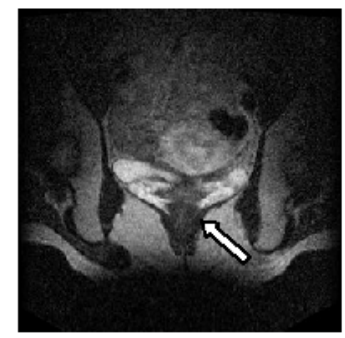

(b)

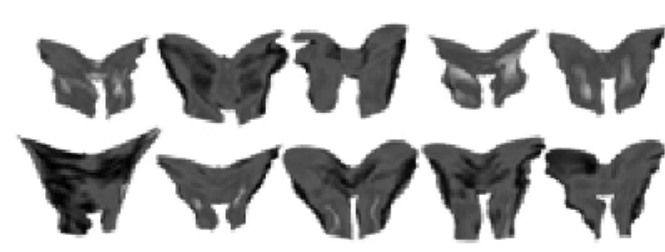

(c)

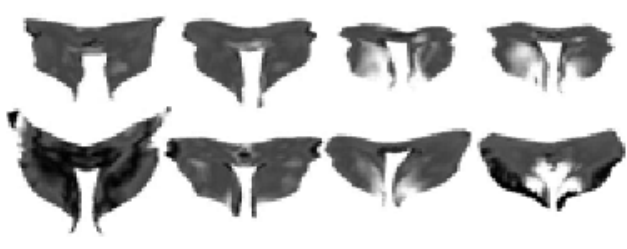

(d)

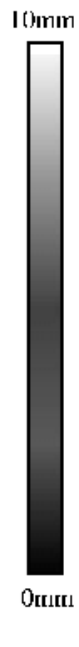

Fig. 2. Example magnetic resonance images from (a) the closed access scanner and (b) the open access scanner and their respective training sets with thickness representation as shown on the scale to the right: (c) the ten subjects and (d) the four subjects in two positions. The levator ani in (a) and (b) is indicated by the white arrows.

The corresponding results by using the proposed combined shape and thickness modelling after principal components analysis is shown in Figure 3. The purpose of the first training set is to depict detailed morphological variations across different subjects by using relatively high SNR images. It is evident from the modelling results that the first mode of variation of the proposed modelling scheme captures the thickness variation in the anterior part of the levator along with a change in position of the levator arms while the second mode shows a slight change in the right section of the levator in both shape and thickness. In the second set showing the dynamic information of the levators during stress, the first mode displays a change in the shape of the levator arms and a change in height of the posterior of the levator and the second mode shows a shape and thickness variation in the anterior of the levator ani. These modes correspond to the shape and thickness change in the levator during

${ }^{1}$ A colour version of this paper can be found at http://www.doc.ic.ac.uk/ gzy/PFM 
squeezing where the levator is under stress. In this case, the levator arms flatten out, the posterior plate of the levator changes angle and it is expected that the thickness shifts accordingly. In both sets, the third mode of variation shows little variation in both shape and thickness.

Figure 4 shows a comparison of the accumulative variance of the statistical models from the parameterization based on shape information only and the parameterization based on both shape and thickness information combined, the proposed modelling technique. As expected, the compactness of the combined technique is less than that of the shape only model due to the increased information dimensionality in the optimization.

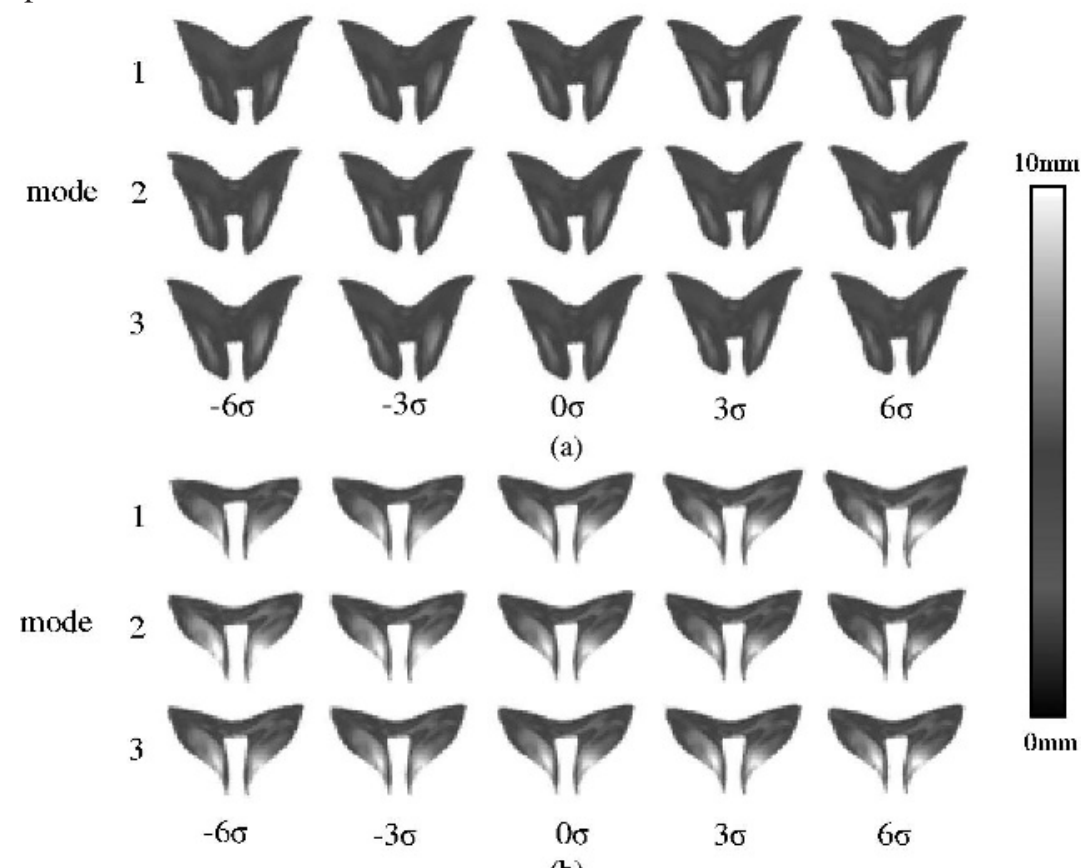

(b)

Fig. 3. Principal components analysis showing the first three modes of variation of the levator ani shapes and corresponding thicknesses of the training set built from images acquired in (a) the closed access scanner to assess shape and thickness variation across subjects at rest, and (b) the open access scanner to assess shape and thickness variation during stress. The thickness color map used can be seen on the right.

\section{Conclusion}

In conclusion, we have described the use of a statistical shape model that includes both surface and thickness information of the levator ani. The objective function used to select the parameterisation of the shape model was based on the MDL criterion. The work extends our previous research on optimal statistical shape modelling for volumetric analysis of the levator ani. With harmonic embedding, it provides an 


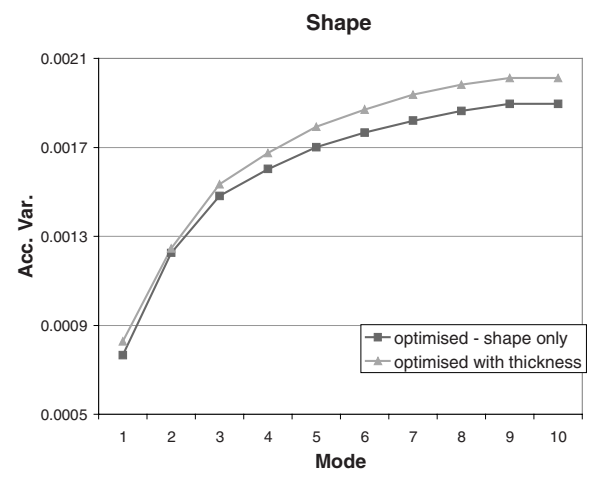

(a)

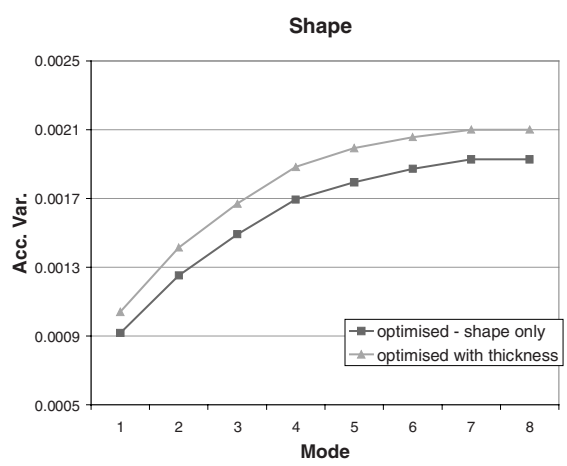

(b)

Fig. 4. Accumulative variance of the shape from the training sets built from images acquired in (a) the closed access scanner and (b) the open access scanner.

effective framework for combining shape with tensor distribution, and is particularly suited for modelling sheet muscles such as the levator ani. Principal component analysis of the derived model has shown meaningful shape and thickening variation of the levator ani, demonstrating the practical value of the technique.

Acknowledgements. The authors would like to thank W. Caspersz at St Mary's Hospital and S. Masood, L. Crowe and D. Firmin at the Royal Brompton Hospital for their assistance in MR data acquisition.

\section{References}

1. Healy, J.C., Halligan, S., Reznek, R.H., Watson, S., Phillips, R.K., Armstrong, P. Patterns of Prolapse in Women with Symptoms of Pelvic Floor Weakness: Assessment with MR Imaging. Radiology, Vol. 203. (1997) 77-81.

2. Law, P.A., Danin, J.C., Lamb, G.M., Regan, L., Darzi, A., Gedroyc, W.M. Dynamic Imaging of the Pelvic Floor Using an Open-Configuration Magnetic Resonance Scanner. Journal of Magnetic Resonance Imaging, Vol. 13. (2001) 923-929.

3. Fielding, J.R., Hoyte, L., Schierlitz, L. MR Imaging of Pelvic Floor Relaxation. Journal of Women's Imaging, Vol. 2. (2000) 82-87.

4. Hoyte, L., Schierlitz, L., Zou, K., Flesh, G., Fielding, J.R. Two- and 3-dimensional MRI comparison of levator ani structure, volume, and integrity in women with stress incontinence and prolapse. American Journal of Obstetrics and Gynecology, Vol. 185. (2001) 11-19.

5. Duchinela, K., Ryhammer, A., Nagel, L.L., Djurhuus, J.C., Constantinou, C.E. MRI of the Aging Female Pelvic Floor; A 3D Perspective of its Principal Organization. International Continence Society Congress, Seoul. (2001)

6. Lee, S-L., Horkaew, P., Darzi, A., Yang, G-Z. Optimal Scan Planning with Statistical Shape Modelling of the Levator Ani. MICCAI (2003). 714-721.

7. Horkaew, P., Yang, G-Z. Optimal Deformable Surface Models for 3D Medical Image Analysis. IPMI (2003). 
8. Hoyte, L., Jakab, M., Warfield, S., Paquette, R., Flesh, G., Zou, K., Fielding, J. Comparison of Levator Ani Thickness in Symptomatic and Asymptomatic Women Using MR-Based 3D Color Mapping. American Urogynecologic Society Scientific Meeting (2003).

9. Pizer, S.M., Gerig, G., Joshi, S., Aylward, S.R. Multiscale Medial Shape-Based Analysis of Image Objects. Proceedings of the IEEE, Vol. 91, No. 10. (2003) 1670-1679.

10. Kanai, T., Suzuki, H., Kimura, F. Three-dimensional geometric metamorphosis based on harmonic maps. The Visual Computer, Vol. 14. (1998) 166-176.

11. Besl, P.J., McKay, N.D. A Method for Registration of 3-D Shapes. IEEE Transactions on Pattern Analysis and Machine Intelligence, Vol. 14. (1992) 239-256.

12. Hoppe, H., DeRose, T., Duchamp, T., McDonald, T., Stuetzle, W. Surface Reconstruction from Unorganized Points. ACM SIGGRAPH (1992) 71-78.

13. Press, W.H., Teukolsky, S.A., Vetterling, W.T., Flannery, B.P. Numerical Recipes in C, $2^{\text {nd }}$ edn. Cambridge University Press (1996) ISBN 0-521-43108-5. 